\title{
Wissenschaft regieren - Eine diskursanalytische Studie zum Forschungsrating des Wissenschaftsrates
}

\author{
Von Christian Baier
}

Zusammenfassung: Der Beitrag präsentiert Ergebnisse einer diskursanalytischen Studie zum Forschungsrating des Wissenschaftsrates, bei der die vom Wissenschaftsrat veröffentlichten einschlägigen Texte analysiert wurden. Das Forschungsrating wird als Praxis des Regierens von Wissenschaft interpretiert, die auf eine systematische Restrukturierung der Selbstwahrnehmung von Wissenschaftlern und akademischen Institutionen zielt und damit auf eine Neujustierung wissenschaftlicher Selbststeuerung hinausläuft. Dabei werden neue Gütekriterien wie Effizienz, Differenzierung, Internationalität und standardisierte Erfassbarkeit in die Wissenschaft eingebracht. Die neue Regierungspraxis setzt sowohl die Technik des Panopticon als auch Verfahren der Normalisierung ein. In Anknüpfung an aktuelle Diagnosen, die auf den ,akademischen Kapitalismus“ und die „unternehmerische Universität" abstellen, werden hier zwei komplementäre Prozesse herausgearbeitet: Erst Normalisierung macht Forschung kalkulierbar, bilanzierbar und damit ökonomisierbar; erst die Ausstattung mit Akteursqualitäten ermöglicht es der Universität, unternehmerisch zu handeln.

\section{Einleitung}

Im Mittelpunkt der vorliegenden Untersuchung steht das Forschungsrating des Wissenschaftsrates. ${ }^{1}$ Darin werden Leistungen von Universitäten und außeruniversitären Forschungseinrichtungen in den Kategorien „Forschungsqualität“, „Effizienz“, „Effektivität“, „Nachwuchsförderung“, „Wissensverbreitung in der Öffentlichkeit“ und „Wissenstransfer in die Praxis“ von Fachgutachtern auf der Basis quantitativer und qualitativer Daten öffentlich bewertet (Wissenschaftsrat 2004). Eine Pilotstudie für die Fächer Chemie und Soziologie wurde bereits 2008 abgeschlossen; eine weitere Studie zur Elektro- und Informationstechnik wird aktuell durchgeführt, eine Ausweitung auf geisteswissenschaftliche Fächer ist in Vorbereitung.

In Politik und Öffentlichkeit erscheint das Forschungsrating als unscheinbares GovernanceInstrument, das zur Verbesserung wissenschaftlicher Selbstkontrolle beitragen soll. In der Soziologie hingegen ist es umstritten; es finden sich sowohl positive Einschätzungen, die das Rating als notwendiges Rationalisierungsinstrument betrachten (Neidhardt 2006 a, 2006 b; Gerhards / Wagner 2008) sowie kritische Beiträge, die auf mögliche Gefahren des Ratings und vergleichbarer Verfahren hinweisen (z.B. Laudel / Gläser 2006; Münch 2009; Münch / Baier 2009).

Ziel des Beitrags ist es, diese Diskussion durch eine kritische Analyse des Diskurses, der im Rahmen des Forschungsratings produziert wird, voranzutreiben. Kritisch ist die Analyse insofern, als sie darauf abzielt, dem Forschungsrating seine diskursiv erzeugte Selbstverständlichkeit zu entziehen, es in all seiner Kontingenz darzustellen und so einer möglichst offenen Diskussion zugänglich zu machen. ${ }^{2}$ Dazu wird zunächst eine theoretische Position entwickelt, die sich auf die Arbeiten Michel Foucaults und der governmentality studies sowie auf Jürgen Links Theorie des Normalismus stützt. Diese theoretische Fundierung ist aus zwei Gründen

1 Für kritische Anregungen danke ich insbesondere Tomas Marttila, Nilgun Massih-Tehrani, Jan Riebling sowie den Gutachtern.

2 Damit unterscheidet sich der Impetus des vorliegenden Beitrags deutlich von der von Riordan, Ganser und Wolbring (2011) vorgelegten Analyse, die von einer nicht weiter zu hinterfragenden Akzeptanz von Evaluationsverfahren in der Wissenschaft ausgeht und das Forschungsrating als ,gegebenes Faktum" betrachtet.

Soziale Welt 62 (2011), S. $371-390$ 
hilfreich: Erstens ist sie anschlussfähig an eine diskursanalytische Methodologie, die mittels eines epistemologischen Bruchs soziale Phänomene ihrer Selbstverständlichkeit beraubt und so ihre Dekonstruktion und theoretisch informierte Rekonstruktion ermöglicht (siehe Abschnitt 2). Zweitens operiert sie mit dem Foucaultschen Begriff des Regierens, der sowohl Fremd- als auch Selbstregierung, sowohl Regierung durch materielle Einflussnahme als auch Regierung durch Rekonfiguration von Selbst- und Weltbildern umfasst und daher geeignet ist, die spezifische Machtlogik von Bewertungsverfahren in der Wissenschaft zu erfassen. Die Leitfrage der Untersuchung lautet: Inwiefern lässt sich das Forschungsrating als eine neue Form des Regierens von Wissenschaft deuten, bei der es darum geht, den Blick der Wissenschaft auf sich selbst und damit die Kriterien ihrer Selbststeuerung zu verändern? Um diese Frage zu beantworten, wird der Diskurs ${ }^{3}$ untersucht, in dessen Rahmen der Wissenschaftsrat als Träger des Forschungsratings das Verfahren und seine Ergebnisse propagiert, erklärt, begründet und deutet.

\section{Methodologische Überlegungen}

Der Begriff „Diskurs“ bezeichnet „eine soziohistorisch spezifische Wissenspraxis, die in einem sozialen Feld, in einem sozialen Bereich anzutreffen ist bzw. war“. Ein Diskurs stellt dabei eine emergente Wirklichkeitsebene dar, ist also als ,überindividuelle Praxis“ zu verstehen, die nach eigenen Regeln funktioniert und nicht auf die Absichten oder Eigenschaften der Teilnehmer des Diskurses reduziert werden kann (Diaz-Bone 2006, 2007). Diskurse können auch als regelhaft strukturierte Systeme von Aussagen beschrieben werden. Einzelne empirisch beobachtbare Aussagen folgen einigermaßen eindeutigen Regeln darüber, was von wem wie wann gesagt werden kann. Diese Regeln bleiben allerdings im Diskurs selbst stets implizit (Foucault 1974). Ziel der Diskursanalyse ist die Rekonstruktion der impliziten Regeln und Prinzipien der diskursiven Praxis.

Die Methode der Diskursanalyse lässt sich allgemein als „reflektierte Rekonstruktion“ bezeichnen (Diaz-Bone 2006). ,Rekonstruktion“ ist dabei ganz wörtlich zu verstehen: Gemeint ist ein Nachbauen des untersuchten Diskurses, allerdings ein kontrolliertes, systematisches, distanziertes Nachbauen, das darauf abzielt, die innere Logik des Gegenstandes in der Rekonstruktion sichtbar werden zu lassen (Barthes 1966). Diese Rekonstruktion mit anderen Mitteln gelingt allerdings nur auf der Basis eines ,epistemologischen Bruchs“ (Diaz-Bone 2007). Die Grundoperation der Diskursanalyse ist damit die Erzeugung eines Bruchs mit der epistemologischen Position des untersuchten Diskurses. Ohne diesen Bruch besteht die Gefahr, in eine reine Nacherzählung des Gegenstandes abzurutschen.

Im vorliegenden Fall bedeutet dies, dass die Analyse aus einer Perspektive zu geschehen hat, die weder mit der Position des Wissenschaftsrates noch mit den bisher in der Literatur vertretenen Positionen zum Forschungsrating zusammenfällt. Die vorliegende Untersuchung bricht mit diesen epistemologischen Positionen, indem sie eine theoriegeleitete Neuperspektivierung vornimmt: Es wird unterstellt, dass der Forschungsrating-Diskurs um die Evaluation deutscher Forschungseinrichtungen ein Diskurs über Bedingungen, Formen, Möglichkeiten und Mittel des guten Regierens der Forschung ist - mithin ein Diskurs, in dem sich Konturen einer neuen Gouvernementalität der Wissenschaft ausmachen lassen. Der Sinn dieser reflektierten Umdeutung besteht in der Neutralisierung der scheinbaren Selbstverständlichkeit, Natürlichkeit und Notwendigkeit des Gegenstandes, die ohne den epistemologischen Bruch den analytischen Blick zu vernebeln droht. Das erzeugte Kontingenzbewusstsein ermöglicht eine kritische Auseinandersetzung mit dem Forschungsrating und den darin manifestierten Formen

3 Im Unterschied zu anderen Diskursanalysen wird hier ein monopolarer Diskurs untersucht, mit dem Wissenschaftsrat als einzigem Sprecher. Diese Begrenzung ist gerechtfertigt, weil der Wissenschaftsrat als Träger des Ratings über ein unangefochtenes Deutungsmonopol verfügt. 
von Wissen und Macht, auch wenn dieses sich selbst diskursiv als selbstverständliche ,Natur der Dinge' oder unausweichliche Folge von ,Sachzwängen' darstellt.

Diese methodologische Ausrichtung hat auch Folgen für den Stellenwert der Theorie: Es werden in Folgenden Konzepte und Modelle aus verschiedenen Theoriesträngen aufgenommen und zur Rekonstruktion des Forschungsrating-Diskurses verwendet. Dabei wird jedoch darauf verzichtet, eine explizite Integration der aufgenommenen Theoriestränge zu versuchen - obwohl dies sicherlich möglich und fruchtbar wäre. Hier steht jedoch die kritische Analyse des empirischen Phänomens Forschungsrating mithilfe der herangezogenen Begriffe im Vordergrund.

\section{Diskurse und Praktiken des Regierens als Machtphänomene}

Die Bewertung wissenschaftlicher Leistung im Forschungsrating wird also im Folgenden als eine überindividuelle, diskursiv verfasste „Praxis des Regierens“ interpretiert, das heißt als mehr oder weniger systematischer und reflektierter Versuch, auf das Handeln von Forschern und Universitäten einzuwirken. Dieser weite Begriff des Regierens wurde von Michel Foucault entwickelt (Foucault 1982, 2006) und steht im Zentrum des konzeptuellen Repertoires der governmentality studies (Dean 2009). Der spezifische Machtbegriff, der hier zum Ausdruck kommt, umfasst Phänomene des Regiert-Werdens und der Selbst-Regierung gleichermaßen und definiert jede Praxis als Regieren, die darauf abzielt, Felder möglicher Handlungen und Reaktionen zu strukturieren (Foucault 1982). Ein wichtiger Aspekt des Foucaultschen Machtbegriffes ist die Einsicht, dass moderne Regierungsweisen Macht und Wissen verschränken und in ein Verhältnis der gegenseitigen Steigerung bringen. Die Macht-Technik der Prüfung koppelt beispielsweise die objektivierende Deskription und die subjektivierende Sanktion von Leistung aneinander. Auf diese Weise wird Wissen über die Kandidaten erzeugt und zugleich werden Anreize geschaffen, die das Feld ihres zukünftigen Handelns restrukturieren (Foucault 2008: 238ff). Die Foucaultsche Perspektive kann also zeigen, wie Wissensproduktion und Machtausübung im Forschungsrating miteinander kombiniert werden. Um die Analyse von Regierungspraktiken zu systematisieren, ist es hilfreich, sich zunächst an einzelnen Dimensionen des Regierens zu orientieren. Dean (2009) schlägt dazu vier Dimensionen vor.

Formen der Sichtbarkeit: Ein Charakteristikum von Regierungspraktiken ist die Art und Weise, in der sie sich den zu regierenden Gegenstandsbereich erschließen. Um auf das Handeln von Menschen in systematischer Weise einwirken zu können, muss zunächst sichergestellt werden, dass der betreffende Bereich der Gesellschaft eindeutig wahrnehmbar, erfassbar, sichtbar ist (Dean 2009: 30 f). Grundsätzlich stellen sich in Bezug auf die Sichtbarkeit drei Fragen: Wie wird das betreffende Feld der Sichtbarkeit konstruiert? Was wird sichtbar gemacht, was bleibt verborgen? Wie wird der zu regierende Bereich repräsentiert?

Rationalitäten: Praktiken des Regierens beinhalten eine je eigene Form von Rationalität, die den kognitiven und diskursiven Rahmen aufspannt, innerhalb dessen Probleme und Ziele definiert werden und Begründung, Planung und Kritik stattfinden können. Dean (1995) bezeichnet diese Dimension von Regierungspraktiken als „episteme of government“"

Techniken: Weiter lassen sich Regierungspraktiken auch durch die Techniken charakterisieren, die sie einsetzen, um innerhalb ihres Interventionsfeldes Wirkungen zu erzielen. Techniken sind diejenigen Elemente, die Rationalitäten, Programme und Ziele in die Praxis umsetzen, also die Verfahren, Prozeduren, Methoden, Apparate und Taktiken, mit denen Regierungspraktiken ihre Objekte identifizieren, Regierbarkeit sicherstellen und Einfluss ausüben (Dean 1996).

Subjekte: Die vierte Dimension betrachtet Formen individueller und kollektiver Identität, die im Rahmen von Regierungspraktiken eine Rolle spielen. In der Literatur wird dieser The- 
menkomplex unter verschiedenen Rubriken behandelt; Identität, Individuum und Subjekt sind die am häufigsten verwendeten Begriffe. Der vorliegende Beitrag verwendet durchgängig die Begriffe „Subjekt“ bzw. „Subjekt-Form“.

\section{1 Normalismus als Element moderner Regierungspraktiken}

Untersuchungen moderner Diskurse und Regierungspraktiken stoßen in den verschiedensten Zusammenhängen auf die Themen Normalität und Normalisierung sowie auf die zugehörige Rationalität, die Jürgen Link als „Normalismus“ bezeichnet (Foucault 2008: 220ff; Ewald 1990; Link 2009). Es handelt sich dabei offenbar um „diskurstragende Kategorien“ (Jäger / Jäger 2007: 61), um Konzepte also, die für den Verlauf und die Funktionsweise von Diskursen in modernen Gesellschaften von größter Bedeutung sind. Ein Ziel der vorliegenden Analyse ist es zu zeigen, dass auch das Forschungsrating Elemente des Normalismus enthält.

Jürgen Link (2009) unterscheidet zwei Typen des Normalismus: „Proto-Normalismus“ zeichnet sich dadurch aus, dass er soziale Phänomene anhand starrer Maßstäbe beurteilt und jede Abweichung durch disziplinierende Sanktionen ahndet und damit den Stillstand sozialer Dynamik im betreffenden Bereich zum Ziel hat. „Flexibler Normalismus“ zielt hingegen nicht auf Stagnation, sondern auf die Förderung von Konkurrenz um „Normalwerte“ und Kanalisierung sozialen Wandels innerhalb normaler Bahnen, sodass weder Stagnation noch sprunghafte Entwicklungen den angestrebten Fortschritt stören. Normalisierung findet dann statt, sobald Ergebnisse einer zunächst deskriptiven Untersuchung eines sozialen Phänomens im Rahmen von Regierungspraktiken präskriptiv gewendet und zur Norm erhoben werden.

Das Forschungsrating beurteilt wissenschaftliche Leistung nicht anhand vordefinierter Schwellenwerte, sondern erfasst das gesamte empirische Spektrum, um ex post abzuleiten, welche Leistungen normal sind und welche dementsprechend als exzellent zu loben oder als unbefriedigend zu tadeln sind. Damit ist es in seiner Ausrichtung eher dem flexiblen Normalismus zuzurechnen; jedoch sind auch deutliche protonormalistische Elemente vorhanden, etwa die Versuche einer exakten Eingrenzung von Disziplinen, die Verwendung der panoptischen Sichtbarkeitstechnik und die Sanktion von Heterogenität und Ambivalenz im Rahmen standardisierter Datenerhebungstechniken (siehe Abschnitt 5).

Wie sich flexibler Normalismus auf den vier Dimensionen der Regierungspraxis jeweils niederschlagen kann, sei kurz angedeutet: Normalistische Sichtbarkeit verwendet Diagramme, Zahlen, Anteils- und Mittelwerte und Rekorde, um soziale Bereiche zu beschreiben, die in ihren Blick geraten. Von symbolisch generalisierter Plausibilität sind dabei der Mittelwert, die Gauß-Verteilung, das lineare Wachstum und der Rekord. Normalismus gewinnt Problematisierungen aus dem Vergleich empirischer Sachverhalte mit diesen symbolischen Idealformen (Link 2009: 340ff). Normalistische Rationalität zielt darauf, eine kontrollierte Wachstumsund Fortschrittsdynamik zu erzeugen, die die Balance hält zwischen sprunghaft hyperdynamischem Wachstum auf der einen und Stagnation bzw. Verfall auf der anderen Seite (Link 2009: 324). Normalistische Techniken dienen dazu, soziale Bereiche ihrer Heterogenität und Komplexität zu entkleiden und sie in leicht regierbare, homogene Normalfelder zu verwandeln, die von quantifizierbaren Leistungsdimensionen aufgespannt werden. Dabei kommen insbesondere Verfahren der standardisierten Datenerhebung und statistischen Kalkulation zum Einsatz (Link 2009: 332ff). Die normalistischen Subjekte schließlich sind „soziale Atome“, äquifunktionale Einheiten, die sich voneinander nur hinsichtlich ihrer Position auf den Dimensionen des Normalfeldes unterscheiden. Im Rahmen normalistischer Regierungsinterventionen werden die Subjekte zu Konkurrenzen um die Erreichung bestimmter Normalwerte (Mittelwert, Rekord) motiviert, woraus letztlich die angestrebte normalisierte Wachstumsdynamik resultiert (Link 2009: 352). 


\section{2 Die panoptische Regierungstechnik}

Neben normalistischen Zügen zeigt das Forschungsrating auch Affinität zu der von Michel Foucault analysierten Machttechnik des Panopticon. Dabei handelt es sich zunächst um ein Modell für ein Gefängnis, in dem die Gefangenen immer fürchten müssen, gesehen und überwacht zu werden, ohne sich dessen jemals sicher sein zu können. Hierin sieht Foucault eine spezielle Machttechnik verkörpert, die sich der Fähigkeit der Subjekte zur Selbstregierung bedient, um diese zu unterwerfen. Die Verschränkung von Macht und Wissen erreicht im Panopticon einen Höhepunkt: Allein die Tatsache, dass die Subjekte sich selbst als Objekte eines unausweichlichen Blicks, eines unabhängigen, unkontrollierbaren Wissens betrachten müssen, sorgt dafür, dass sie sich diesem Blick selbständig unterwerfen, zum „Prinzip der eigenen Unterwerfung werden“ (Foucault 2008: 260). Auf diese Weise kann es gelingen, Machtwirkungen $\mathrm{zu}$ entindividualisieren und $\mathrm{zu}$ automatisieren, sodass sich die erzielten Machteffekte nicht hemmend, sondern gerade steigernd auf die Produktivität und Funktionalität der Beherrschten auswirken. Die dem Panopticon zugrunde liegende Formation kann laut Foucault in einer Vielzahl anderer Bereiche eingesetzt werden und überall dazu dienen, Subjekte zur kontrollierten Selbstregierung anzuleiten (Foucault 2008: 251-294).

Die Analyse wird zeigen, dass das Forschungsrating auf die Installation einer panoptischen Struktur von Wissensbeziehungen in der Wissenschaft zielt. Allerdings ist auch der inzwischen eingeführte komplementäre Begriff des „Synopticons“ von einiger Relevanz: Während das Panopticon Wenigen ermöglicht, Viele zu sehen, erlaubt die Technik des Synopticons Vielen den Blick auf Wenige - etwa im Rahmen massenmedialer Darstellungen (Mathiesen 1997: 217ff). Mithilfe Mathiesens Erweiterung des Foucaultschen Modells lässt sich erkennen, dass die Sichtbarkeitsdispositive moderner Gesellschaften durch Verschränkungen panoptischer und synoptischer Techniken gekennzeichnet sind. Im Falle des Forschungsratings eröffnet zunächst eine panoptische Struktur das gesamte Spektrum der Wissenschaft einem unausweichlichen Blick; darüber hinaus aber wird dieser neu etablierte Blickpunkt mittels synoptischer Techniken einem breiten öffentlichen Publikum zugänglich gemacht. Wenn im Folgenden die panoptische Dimension des Forschungsratings im Vordergrund steht, so deshalb, weil seine Wirkung auf die Wissenschaft nicht davon abhängig ist, ob sich tatsächlich eine breite Öffentlichkeit für die produzierten Leistungsmessungen interessiert. Die Analyse wird zeigen, dass der Diskurs selbst eine Reihe von Subjekten beschreibt, die aus verschiedenen Gründen an einer Bewertung der Wissenschaft interessiert sind. Jedoch bleibt dieses im Diskurs evozierte Publikum für die bewerteten Einrichtungen und Wissenschaftler stets unsichtbar und es besteht keine Rückmeldung darüber, ob und von wem die bereitgestellten Bewertungen tatsächlich rezipiert werden. Die Effekte des Ratings auf die wissenschaftliche Praxis basieren damit nicht so sehr auf seiner Rezeption im Rahmen synoptischer Dispositive, sondern auf dem neu eingerichteten Blickpunkt selbst, der neue, unausweichliche Sichtbarkeit schafft und die Gesehenen damit zu spezifischen Formen der Selbstregierung motiviert.

\section{Methodik}

Im Rückgriff auf die leitende Fragestellung fällt es leicht, einen Textkorpus zusammenzustellen und die gewählte Zusammenstellung zu begründen. Die Materialsammlung stützt sich auf das ,theoretisches Sampling“ (Strauss / Corbin 1999), das mit einer diskursanalytischen Methodik kompatibel ist (Schwab-Trapp 2004). Da hier der Diskurs des Forschungsratings untersucht werden soll, kann der Korpus auf jene Texte begrenzt werden, die von offizieller Seite über das Forschungsrating sprechen, nämlich auf die Gesamtheit der vom Wissenschaftsrat veröffentlichten Texte zum Forschungsrating.

Die verwendete Codierstrategie erschließt sich ihren Gegenstand von zwei Seiten, indem sie einerseits für die Eigenheiten des Gegenstandes möglichst sensitiv bleibt, sich dabei aber 
durch die bisher entwickelten theoretischen Überlegungen leiten lässt. Die Theorie dient dabei zur Entwicklung von Heuristiken, die Hinweise darauf geben, worauf es sich zu achten lohnt. Zur technischen Umsetzung der Datenanalyse wurde das Softwarepaket ATLAS.ti verwendet (vgl. Diaz-Bone / Schneider 2004). Die Texte wurden von der Website des Wissenschaftsrates heruntergeladen, lagen also in digitaler Form vor. Der Textkorpus umfasste 7 Dokumente mit insgesamt 509 Druckseiten. Als Vorbereitung auf die Datenanalyse wurden alle Texte zunächst einmal gelesen. Daran schloss sich der computergestützte Codierprozess an. Insgesamt wurden 41 Codes verwendet, 573 Textstellen wurden codiert.

\section{Der Diskurs des Forschungsratings}

Die folgenden Abschnitte präsentieren die Ergebnisse der Analyse. Die Darstellung verläuft dabei entlang der oben eingeführten vier Dimensionen von Regierungspraxis. Eine umfassende Beschreibung der im Forschungsrating verwendeten Verfahren kann hier nicht geleistet werden; der Schwerpunkt liegt auf der diskursanalytischen Rekonstruktion, weshalb auf eine nacherzählende Wiedergabe der Textinhalte weitgehend verzichtet werden muss.

\section{1 Sichtbarkeit: Bilder einer Forschungslandschaft}

Das Forschungsrating repräsentiert die Forschung der deutschen Chemie und Soziologie mithilfe dreier Sichtbarkeitsformen, die im Folgenden als „Panorama“, „Porträt“ und „Röntgenbild" bezeichnet werden (Abb.1). ${ }^{4}$

Das Panorama (Abb.1 links oben) nimmt den gesamten sozialen Bereich in den Blick, auf den sich das Forschungsrating bezieht; es ist ein Bild des jeweiligen Faches in seiner Gesamtheit. Die Funktion des Panoramas entspricht der üblichen Verwendungsweise dieses Bildtyps: Es geht darum, die Charakteristika einer Landschaft - hier der deutschen Forschungslandschaft - darzustellen, indem man sie dem Betrachter auf einen Blick zugänglich macht. Das Panorama dient als Gesamtschau aller relevanten Einheiten und Dimensionen, es ist zugleich Zusammenfassung, Überblick und Hintergrund weiterer Aussagen und Repräsentationen.

Das Panorama folgt einer normalistischen Logik der Sichtbarmachung, indem es die Verteilungen wissenschaftlicher Leistung in assoziative Nähe zur Gaußschen Normalverteilung bringt. Diese Darstellungsform ist keineswegs zufällig, denn dieselben Sachverhalte ließen sich auch mithilfe anderer Diagrammtypen repräsentieren, etwa in Kreisdiagrammen, wodurch allerdings kein Bezug zur Normalverteilung entstünde. Für eine Regierungspraxis ist es aus zwei Gründen günstig, die Bewertungsergebnisse auf diese Weise darzustellen: Die Normalverteilung ist erstens ein normalistisches Kollektivsymbol von großer Bedeutung, weshalb die gewählte Darstellung dazu führt, dass die symbolisch generalisierte Plausibilität der Normalverteilung zumindest teilweise auf die Ergebnisse übergeht. So gewinnen die Bewertungsergebnisse an Glaubwürdigkeit - sie , sehen gut aus'. Zweitens lenkt diese Sichtbarkeitsform den Blick auf Abweichungen von der implizierten glatten Normal-Kurve, auf Brüche der homogenen Form, und rückt diese in den Mittelpunkt der Aufmerksamkeit. Das Panorama zeigt besonders deutlich die Gipfel, aber auch die Schluchten der Forschungslandschaft und liefert damit Anreize und Orientierung für normalisierende Eingriffe. Die Tatsache etwa, dass in der deutschen Soziologie mehr negative als positive Abweichungen vom Leistungsdurchschnitt existieren (Abb.1 links oben), wird schon durch die Sichtbarkeitsform selbst als Problem konstruiert und definiert - genauer gesagt durch den impliziten Vergleich mit der glatten GaußKurve, der in die Sichtbarkeitsform des Panoramas eingebaut ist.

4 Diese Begriffe entstammen nicht den analysierten Dokumenten, sondern bezeichnen entsprechend der Luhmannschen Logik der Beobachtung zweiter Ordnung die Art und Weise, wie das Forschungsrating seine Umwelt beobachtet und repräsentiert. 


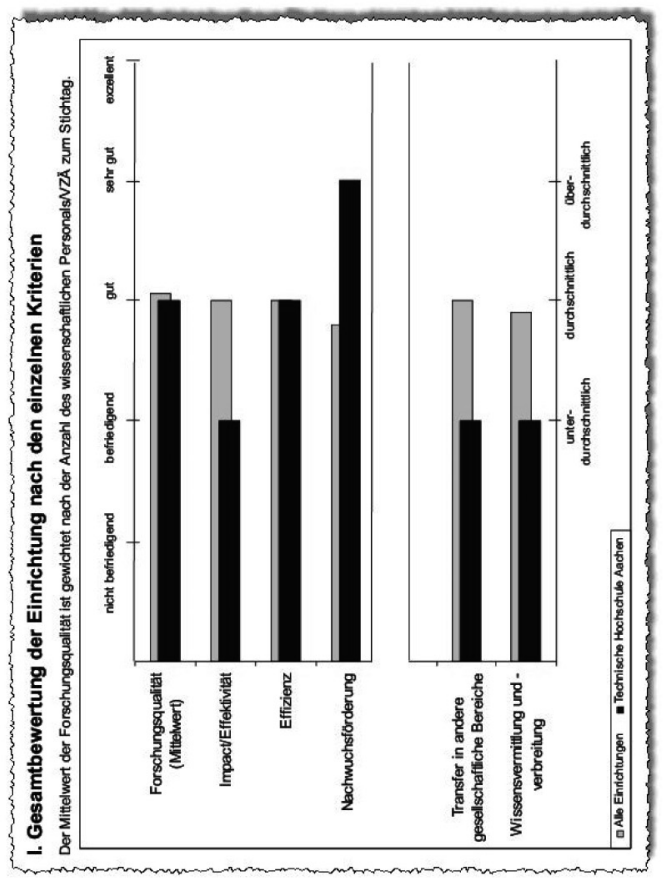

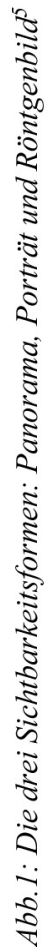
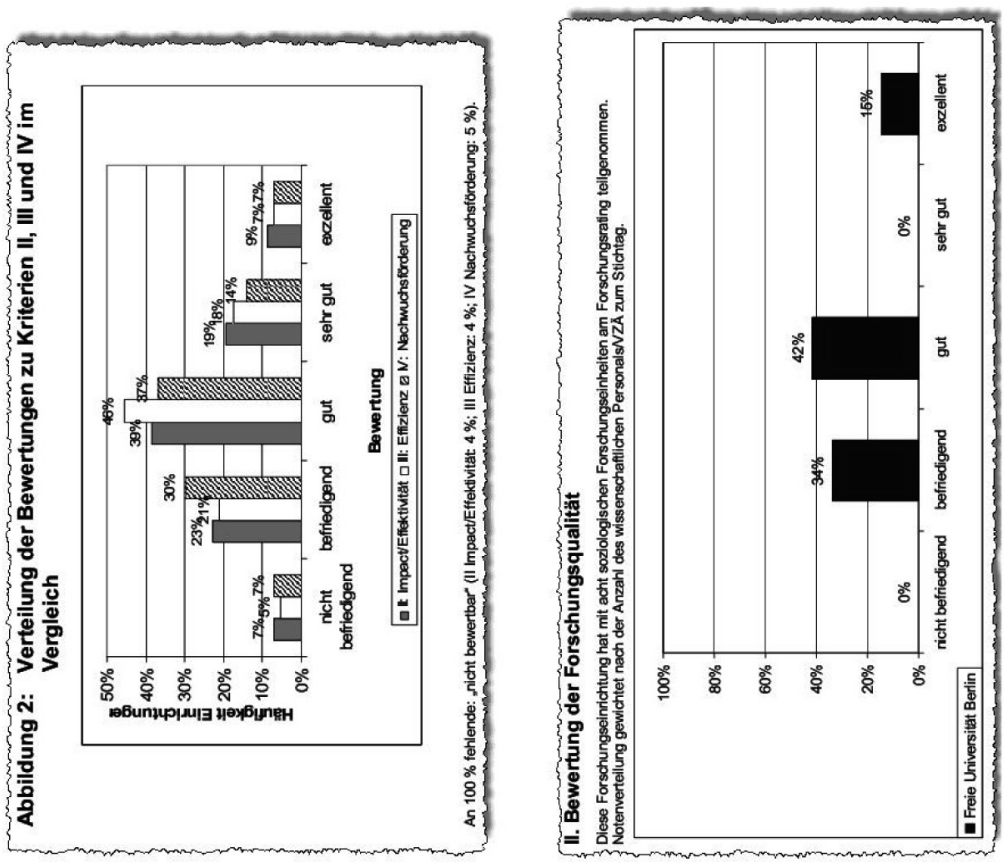

5 Die Bilder sind dem Bericht der Bewertungsgruppe Soziologie entnommen (S. 28, 45, 49) 
Die zweite Sichtbarkeitsform ist das „Porträt“, das einzelne Forschungseinrichtungen zeigt (Abb. 1 rechts). Die Forschungseinrichtungen spielen im Forschungsrating eine besondere Rolle, sie werden als organisationsförmige Quasi-Akteure konzipiert, ihnen werden eigene Motive, Strategien und ,Persönlichkeits'-Eigenschaften zugeschrieben (siehe Abschnitt 5.4). Ein Hauptergebnis des Ratings ist daher die Porträtierung der Einrichtungen. Mit Hilfe der Porträts sollen die ,Charakterzüge' der Einrichtungen, ihre „Stärken und Schwächen“ sichtbar gemacht werden:

„Diese ermöglicht es, Einrichtungen mit unterschiedlichen Missionen nach gleichen Maßstäben zu bewerten und ihnen ein individuelles Profil ihrer Stärken und Schwächen aufzuzeigen. " (Bewertungsgruppe Chemie: 5)

Auch diese zweite Form der Sichtbarkeit weist normalistische Züge auf: Es geht um die Herstellung von Vergleichbarkeit zwischen Einrichtungen, die man unter anderen Umständen als grundverschieden betrachten müsste. Trotz unterschiedlicher „Missionen“ werden die Einrichtungen als Elemente eines Normalfelds porträtiert, das durch einheitliche Dimensionen nämlich die Leistungskriterien des Ratings - aufgespannt wird. Qualitative Unterschiede, die für mangelnde Kommensurabilität sprechen, werden im Rahmen der Porträtierung eingeebnet. Eine genauere Betrachtung des Porträts liefert tiefere Einblicke in die Funktionslogik dieser Sichtbarkeitsform: Jeder Bewertungsdimension sind zwei Balken zugeordnet; der erste zeigt den Wert der porträtierten Einrichtung, der zweite den Mittelwert aller Einrichtungen. Die einzelne Einrichtung wird also nicht durch die von ihr erzielten Bewertungen charakterisiert, sondern durch die Abstände zwischen den individuell erzielten und den durchschnittlichen Bewertungen. Auch das Porträt fokussiert also die Abweichung von einem Normalwert, einem Normal-Porträt, das ex post anhand des Mittelwertes definiert wird. Im Normalporträt treten jene Verteilungen in kondensierter Form wieder auf, die im Panorama in entfalteter Form die Forschungslandschaft aufspannen - die Sichtbarkeitsformen und Normalwerte verweisen aufeinander, stützen einander, gehen ineinander über. Diese Kopplung macht deutlich, dass Sichtbarkeitsformen im Diskurs des Forschungsratings nicht disparat nebeneinander stehen, sondern einer spezifischen Strategie der Sichtbarmachung folgen. Ein Hauptergebnis des Forschungsratings ist damit ein Arrangement von Bildern, das die Forschungseinrichtungen in systematischer Form aus verschiedenen Perspektiven darstellt und gleichzeitig mit Normalisierungsimperativen umstellt. Denn auch hier wirkt die Fokussierung der Abweichungen darauf hin, schwächere Einrichtungen zur Selbstnormalisierung anzuregen und Spitzenreiter zur Konkurrenz um sichtbare Rekorde zu motivieren.

Die dritte Komponente in diesem Sichtbarkeitssystem ist das „Röntgenbild“(Abb. 1 unten). Es zeigt die Verteilung des Kriteriums „Forschungsqualität“ über die einzelnen Forschungseinheiten, aus denen sich die dargestellte Einrichtung zusammensetzt. Als Sichtbarkeitsform erfüllt das Röntgenbild die Funktion einer Innenansicht, gewährt Einblick in das Innenleben der Einrichtungen und erschließt damit ein weiteres Feld möglicher Interventionen. Der Diskurs um diese dritte Sichtbarkeitsform macht deutlich, wie sehr das Forschungsrating um eine möglichst tief greifende Sichtbarmachung seines Objektbereichs bemüht ist: Wie in Abb. 1 zu sehen ist, bleiben in den Röntgenbildern die Einheiten selbst anonym, d.h. es ist nicht ersichtlich, welcher Lehrstuhl bzw. welche Forschergruppe wie bewertet wurde. Diese Anonymisierung erfolgt aufgrund datenschutzrechtlicher Bedenken, die allerdings im Diskurs kritisch kommentiert werden:

„Es sollte künftig unbedingt möglich sein, die auf der niedrigen Ebene der Forschungseinheiten vorgenommenen Bewertungen der Forschungsqualität auch der Öffentlichkeit zugänglich zu machen. [...] Weil diese Details indes einen hohen Informationsgehalt haben, der möglicherweise auch für externe Adressaten von Interesse sein könnte. " (Bewertungsgruppe Chemie: 55) 
„Eine Veröffentlichung auf Ebene der Forschungseinheiten würde es den Einrichtungen erleichtern, die Ergebnisse im Vergleich mit den jeweils unmittelbaren Konkurrenten [...] zu interpretieren. " (Steuerungsgruppe: 55)

Das Forschungsrating zeigt sich entschlossen, die Reichweite seines Blickes so weit wie möglich auszudehnen. Nicht nur die Forschungslandschaft soll dem externen Blick zugänglich gemacht werden, sondern der Blick und seine Machteffekte sollen auch die einzelne Einrichtung erfassen und bis in ihr Inneres hineinreichen. Spätestens an dieser Stelle wird klar, dass auch das Prinzip des Panopticon die Sichtbarkeitsformen des Forschungsratings prägt: Je tiefer der Blick reicht, je individualisierender er ist, je weiter der Bereich des Wissens ausgedehnt wird, desto weiter reichen auch die Machteffekte des Ratings, desto mehr werden die Subjekte motiviert und befähigt, die bereitgestellten Normalisierungsschablonen zur Selbstregierung zu verwenden. Die Präsenz der panoptischen Machttechnik in den Sichtbarkeitsformen des Ratings wird auch in folgendem Zitat deutlich, das beispielhaft für viele ähnlich Aussagen im Diskurs stehen kann:

„Ziel ist, den Wettbewerb in der Forschung effektiver und effizienter zu machen, indem die Transparenz der Forschungsleistungen im öffentlichen Sektor erhöht wird, und die forschenden Einrichtungen bei ihrer Profilierung im Rahmen ihrer jeweiligen Mission zu unterstützen, indem ihnen durch vergleichende Leistungsbewertungen eine Standortbestimmung nach international gültigen Maßstäben ermöglicht wird. "(Steuerungsgruppe: 12)

Der Diskurs bespricht hier Sichtbarkeit - „Transparenz“ - als eigenständiges Mittel der Disziplinierung: Schon die Sichtbarkeit alleine - nicht erst dadurch ermöglichte Sanktionen - führt demnach zur Effizienzsteigerung, weil Leistungen und Defizite eindeutig zugeordnet und an „,international gültigen Maßstäben“ gemessen werden können. Es wurde bereits gezeigt, dass die Sichtbarkeitsformen des Ratings Inkommensurabilitäten nivellieren und Vergleichsmöglichkeiten vervielfältigen. Normalistische Elemente sind explizit in den Aussagen enthalten, die diese Sichtbarkeitsformen erläutern und begründen. In ähnlicher Weise finden sich Bezüge zum Panopticon nicht nur in der Konfiguration von Blicken und Bildern, sondern werden im Zusammenhang mit Transparenz auch explizit formuliert. Die Sichtbarkeitsformen des Forschungsratings verweisen damit auf die Dimension der episteme of government, die im Folgenden untersucht wird.

\section{2 Rationalität: Das Forschungsrating als panoptisches Interface}

Im Zentrum einer Rationalität des Regierens stehen Problematisierungen bestimmter Eigenschaften des zu regierenden Phänomens. Diese Problematisierungen können entweder explizit formuliert werden oder - wie im Fall des Forschungsratings - implizit in Programmen und Zielen enthalten sein. Eine allgemeine Zielvorgabe findet sich in folgendem Abschnitt:
„Die Anforderungen an die Selbststeuerungsfähigkeiten der Einrichtungen und damit an strategisches Steuerungswissen sind [...] erheblich gestiegen. Gleichzeitig erzeugt die wett- bewerbliche Differenzierung auch einen größeren Bedarf an Orientierungswissen für Stu- dienanfänger, wissenschaftlichen Nachwuchs und Kooperationspartner wissenschaftlicher Einrichtungen. Nicht zuletzt zieht die zunehmende Autonomie wissenschaftlicher Einrich- tungen die Forderung von Politik und Gesellschaft nach mehr Transparenz über die er- brachten Leistungen nach sich. " (Steuerungsgruppe: 9)

Das Forschungsrating möchte „Steuerungswissen“ für die Einrichtungen und „Orientierungswissen“ für wissenschaftsexterne Interessenten bereitstellen. Die Rationalität des Regierens, die hier formuliert wird, beruht primär auf diesen beiden Facetten des produzierten Wissens und ihren jeweiligen Machteffekten. 
Betrachtet man zunächst den Aspekt des Steuerungswissens, so zeigt sich, dass diese Wissensform auf die Einrichtungen selbst zielt und ihnen als Normalisierungsschablone dienen soll. Steuerungswissen signalisiert, wie gut Einrichtungen im Vergleich zueinander und relativ zum Durchschnitt abschneiden. Nach normalistischer Manier bezieht es seine Legitimation nicht aus normativen Vorstellungen jenseits der Wissenschaft, etwa aus gesellschaftlichen Nützlichkeitsstandards, sondern stützt sich allein auf Ergebnisse statistischer Verfahren, die empirisch erhoben und anschließend normativ gedeutet werden. Steuerungswissen wird so zum dynamischen Maßstab für richtiges, erfolgreiches oder normales Handeln und teilt den Einrichtungen anhand dieses Maßstabes Positionen im ,Normalfeld Forschung' zu. Die Selbsteinschätzungen der Einrichtungen werden so an die Normalwerte und damit indirekt aneinander gekoppelt - denn unter der Ägide des Steuerungswissens können sie sich nur noch durch positive Abweichungen vom Normalprofil als Produzenten hochwertiger Forschung begreifen und darstellen. Auf diese Weise werden sie motiviert, negative Abweichungen vom Durchschnitt zu vermeiden und in Konkurrenz zueinander nach Verbesserung der eigenen Position zu streben. Weil flexible Normalitäts-Maßstäbe sich mit jeder Leistungssteigerung ebenfalls nach oben verschieben, bedeutet jeder Fortschritt einer Einrichtung zugleich eine Abwertung für andere; der Wettbewerb um Rekorde und der Kampf gegen Ent-Normalisierung werden auf diese Weise auf Dauer gestellt.

Der zweite, durch die Logik des Panopticon geprägte Aspekt dieser Regierungskunst zeigt sich in der Facette des Orientierungswissens: In ihrer Funktion als Orientierungswissen sollen die Ergebnisse des Ratings Politiker, Studenten, Nachwuchswissenschaftler und potenzielle Kooperationspartner in der Wirtschaft über die Leistungen der Einrichtungen informieren. Ihr Zweck ist es damit, die Beziehungen zwischen den Einrichtungen und wissenschaftsexternen Akteuren und Instanzen zu verändern, genauer, die Einrichtungen zu Objekten eines Wissens zu machen, das jenseits ihrer Kontrolle liegt und verschiedenen externen Akteuren zur Verfügung gestellt wird. Diese Installation neuer, einseitiger Wissensbeziehungen verläuft parallel zur oben skizzierten Umstellung der Einrichtungen mit einem Ensemble von Sichtbarkeitsformen. Indem die Einrichtungen dem Blick eines potentiell interessierten Publikums unterworfen werden, entsteht ein Machteffekt, der dazu führt, dass die Einrichtungen den Blick von außen antizipierend auf sich selbst richten. Die Funktionsweise dieser Konfiguration lässt sich in Anlehnung an Foucault folgendermaßen beschreiben: Wer dem Wissen anderer unterworfen ist und dies weiß, internalisiert die Erwartungen der Beobachter und wendet sie auf sich selbst an (vgl. Foucault 2008: 260). Das Orientierungswissen ist ein Mittel, die Einrichtungen einem unentrinnbaren Blick von außen auszusetzen und zur vorauseilenden Internalisierung externer Interessen und Maßstäbe zu veranlassen.

Insgesamt sieht die Rationalität des Forschungsratings vor, die soziale Formation der Forschungseinrichtungen durch den Aufbau neuen Wissensbeziehungen zu verändern. Das Rating bringt eine neue Art der sozialen Relationierung und Interaktion in Gang, die durch Wettbewerb entlang vorgegebener Dimensionen zu effizientem Wissensfortschritt führen soll. Harrison Whites Begriff des „Interface“ (White 2008: 80-85) beschreibt treffend die resultierende soziale Formation: Die drohende Sichtbarkeit von außen (Orientierungswissen) motiviert die Einrichtungen zur intensivierten und ständigen gegenseitigen Beobachtung anhand der durch das Rating sichtbar gemachten Eigenschaften (Steuerungswissen). Wie ein halbdurchlässiger Spiegel positioniert das Interface auf der einen Seite die Forschungseinrichtungen, die nur ihr eigenes Bild und das ihrer Konkurrenten vor Augen haben; auf der anderen Seite des Spiegels müssen die Einrichtungen eine nicht näher einzugrenzende Menge kritischer Beobachter vermuten, von deren Urteilen sie abhängig sind. Das Resultat ist eine Intensivierung der gegenseitigen Beobachtung und des Wettbewerbs zwischen den Einrichtungen um möglichst hohe oder zumindest normale Werte auf jenen Bewertungsdimensionen, anhand derer sie von auBerhalb beurteilt werden können. 
Die Herstellung dieser neuen sozialen Formation ist das primäre Ziel des Forschungsratings. Um jedoch die beabsichtigten Ergebnisse zu verwirklichen, muss das Verfahren bestimmte Bedingungen erfüllen, die im Folgenden als „Rationalitätskriterien“ des Forschungsratings bezeichnet werden. Im Diskurs tauchen sie auf, wenn es darum geht, die Nützlichkeit, Verlässlichkeit und Überlegenheit des Forschungsratings herauszustreichen. Die beiden übergeordneten Rationalitätskriterien sind „Wahrheit“ und „Ökonomie“. Wahrheit steht dabei eindeutig im Mittelpunkt, während Fragen der Ökonomie eher am Rande diskutiert werden. Die Bedeutung der Ökonomie im Diskurs zeigt sich vor allem darin, dass Effizienz - die Leitidee sowohl der neoklassichen Ökonomie als auch des New Public Management - im Forschungsrating als Gütekriterium für Forschung verwendet wird. Forschung muss demnach die Relation von Zweck und Mittel optimieren, um exzellent sein zu können. Auf das Kriterium der Wahrheit geht der Diskurs näher ein. Er entwickelt zwei Unterkriterien, die die Wahrheit und Verlässlichkeit des produzierten Wissens garantieren sollen: Internationalität und Differenzierung.

Die Auffassung, dass Internationalität in jeder Beziehung und überall von größter Bedeutung ist, scheint eine der unhinterfragten Grundannahmen im Diskurs zu sein. Eine Regel des Diskurses legt offenbar fest, dass bereits der Verweis auf Internationalität ausreicht, um Zweifel am Bewertungsverfahren oder dessen Ergebnissen auszuräumen. Internationalität fungiert dabei gleichzeitig als zu verwirklichendes Ziel und als Quelle von Legitimation für eine Vielzahl von Entscheidungen:

„Die fünfstufige Bewertungsskala entspricht internationalen Beispielen. “ (Bewertungsgruppe Chemie: 50)

„Angesichts der internationalen Erfahrungen [...] schließt der Wissenschaftsrat eine ausschließlich auf quantitativen Indikatoren basierende Forschungsbewertung [...] aus. " (Empfehlungen zu Rankings: 42f.)

„Die internationale Erfahrung der Gutachter ist gerade in den Fächern, für die internationale Vergleichsdaten nicht vorliegen, besonders wichtig, um eine Bewertung nach internationalen Maßstäben sicherzustellen. " (Steuerungsgruppe: 32)

„Die im Rahmen der Pilotstudie Chemie für die Zitationsanalyse verwendeten Indikatoren entsprechen dem internationalen Stand der bibliometrischen Forschung. " (Steuerungsgruppe: 39)

Gutachter besitzen internationale Erfahrung, die Bewertung arbeitet mit international üblichen Indikatoren, das Verfahren zieht internationale Erfahrungen mit Forschungsevaluation in Betracht und die Bewertungsskala entspricht internationalen Gepflogenheiten: Die diskursive Strategie, die hier hervortritt, speist Internationalität auf allen denkbaren Ebenen in das Bewertungsverfahren ein und verwandelt sie damit in eine Grundlage für die Wahrheit der Bewertungsergebnisse. Die Verknüpfung von Internationalität und Wahrheit ist dabei keineswegs reiner Selbstzweck, sondern Ausdruck einer indirekten Rationalität des Regierens: Über den Umweg einer Selbstbindung verpflichtet das Forschungsrating auch die Forschungseinrichtungen auf Internationalität. Wenn nämlich die Bewertung nach internationalen Maßstäben durch internationale Gutachter erfolgt, müssen die Einrichtungen sich selbst ebenfalls an diesen Maßstäben orientieren, um gute Bewertungen erzielen zu können. Indem also das Rating die Wahrheit seiner Bewertungen an Internationalität knüpft, wird Internationalität zu einem Symbol für hochwertige Forschung verallgemeinert: Internationale Forschung - so könnte man zusammenfassen - ist exzellente Forschung.

Das zweite Kriterium für Wahrheit ist „Differenzierung“, genauer: Differenziertheit der Bewertungsergebnisse und zugleich strategische Differenzierung der „Profile“ und „Missionen“ der Forschungseinheiten. Auch Differenzierung ist ein Kernbegriff, der im Diskurs allem Wünschenswerten und Richtigen beigegeben wird. Wie Internationalität changiert auch Differenzierung zwischen deskriptiver und präskriptiver Bedeutung: 
„, So ist heute anerkannt, dass das Hochschulsystem nur dann sowohl den wachsenden Bedarf an tertiärer Bildung decken als auch international herausragende Forschungsleistungen erbringen [...] kann, wenn die einzelnen Hochschulen Schwerpunkte bilden und sich unterschiedliche Hochschulprofile im Wettbewerb herausdifferenzieren. " (Steuerungsgruppe: 9)

„, Insgesamt führen die sechs Kriterien zu einer sinnvollen Differenzierung der Bewertung, so dass [...] die Bewertungsergebnisse insgesamt stark heterogen sind und damit offenbar das jeweils individuelle Leistungsprofil der einzelnen Einrichtungen adäquat erfassen. " (Bewertungsgruppe Chemie: 53)

Das Forschungsrating erklärt im ersten Zitat die Produktion von Differenzierung unter den Einrichtungen zu einem seiner Ziele, argumentiert im zweiten Zitat aber, als sei die angestrebte Differenzierung bereits verwirklicht und daher als Prüfkriterium für die Wahrheit der Bewertung geeignet. Diese begriffliche Unschärfe verknüpft die Frage nach wissenschaftlicher Leistung unauflöslich mit der Frage nach Differenzierung. Weil diese Verknüpfung eine Grundlage des Bewertungsverfahrens ist, wirkt sie nicht nur als Rhetorik auf Leser der Dokumente; vielmehr wird diese spezifische Form der Differenzierung direkt zu einer relevanten Dimension des Handelns der bewerteten Einrichtungen. Wer gut bewertet werden will, muss mit Differenzierung rechnen, muss Differenzierung als Ziel akzeptieren und Differenzierung nachweisen, denn im Forschungsrating gilt: Differenzierte Forschung ist gute Forschung.

Insgesamt prägt eine Kombination normalistischer und panoptischer Elemente die Rationalität des Forschungsratings. Das bereitgestellte Orientierungs- und Steuerungswissen hat die Aufgabe, die Wissenschaft als homogenes Normalfeld zu repräsentieren sowie Leitkriterien und Normalitätsschablonen zu liefern, vor deren Hintergrund die Einrichtungen in effizienzsteigernde Konkurrenzen eintreten können. Motivierend und kontrollierend sollen dabei die neuen Einblicke wirken, die das Forschungsrating für kritische Beobachter der Wissenschaft bereithält. Das Forschungsrating zielt darauf, die bewerteten Einrichtungen in die spezifische soziale Formation eines Interface einzubinden, in dem sie durch das Wissen um externe Beobachter motiviert in intensivierte Formen gegenseitiger Beobachtung und Konkurrenz eintreten. Mit dem Interface verknüpfen sich hier dieselben Hoffnungen, die laut Foucault auch Bentham mit seinem Panopticon verband: Hoffnung auf eine Technik der Machtausübung, die die Funktionalität der Regierten steigert, statt sie zu hemmen; auf ein formales Regieren auf Distanz, das letztlich ohne Sanktion und Eingriff auskommt, ein ökonomisches, reibungsarmes Regieren durch Verschränkung von Wissen und Macht.

\section{3 Techniken: Normalisierung durch zwangsbewehrte Messverfahren}

Im Rahmen des Forschungsratings kommt eine ganze Reihe von Techniken zum Einsatz, die eigenständige Machteffekte entfalten und daher als Elemente der Regierungspraxis zu betrachten sind. Im Vordergrund stehen dabei Techniken der Datensammlung, Aufbereitung und Bereinigung, statistische Techniken der Kalkulation (etwa von Perzentilen und Mittelwerten) sowie Techniken der Zusammenstellung und Repräsentation von Daten. Das Forschungsrating verwendet als Bewertungsbasis eine ganze Reihe meist quantitativer Daten aus unterschiedlichen Quellen: Bibliometrische Publikations- und Zitationszahlen werden aus Literaturdatenbanken extrahiert; Informationen über Mitarbeiterzahl, Ausstattung, Menge der eingeworbenen Forschungsmittel und weitere Indikatoren werden von den zu bewertenden Einrichtungen eingeholt, auf Vollständigkeit und Validität kontrolliert und aufbereitet; darüber hinaus werden eingereichte Kontextinformationen und Texte kontrolliert und mit den übrigen Daten verbunden. Erst die an sich unscheinbaren Techniken des Zählens, Prüfens, Verrechnens und Vergleichens, die hierbei zum Einsatz kommen, ermöglichen durch die Art ihrer Anwendung den Aufbau des Interface und die Normalisierung der Forschung: Techniken definieren und iso- 
lieren Normaleinheiten, legen die Grenzen und Dimensionen der Normalfelder Chemie und Soziologie fest, rechnen den einzelnen Einrichtungen Leistungen zu und motivieren sie vor allem zu Selbstnormalisierungsmaßnahmen. Erst im Rahmen dieser Techniken erschließt sich das Feld der Sichtbarkeiten, erst durch Abgrenzung und Definition werden die Einheiten und Subjekte definiert, die beim Regieren von Wissenschaft von Bedeutung sind. Damit wird klar, dass die Dimension der Techniken in enger Beziehung zu den Dimensionen der Sichtbarkeit und der Subjekt-Formen steht.

Die technischen Hauptprobleme des Forschungsratings betreffen Fragen der Abgrenzung und Zurechnung, die sich aus der Heterogenität, Vielfalt und Unschärfe seines Gegenstandsbereiches ergeben. Weder die Einheiten, um deren Bewertung es geht, noch die Fächer, denen diese Einrichtungen angehören, sind einfach in der sozialen Realität vorzufinden. Vielmehr müssen sowohl die Fächer als Normalfelder und die Forschungseinrichtungen als Normaleinheiten zunächst aus einer komplexen und heterogenen Wirklichkeit herauspräpariert werden. In der Chemie ist es offenbar schwierig, die Fachgebiete in den Griff zu bekommen:

„,Der erste Schritt der fachspezifischen Operationalisierung war die Definition des Faches Chemie. Die Einteilung in zehn Teilgebiete machte das Fach [...] handhabbar. Allerdings sind die einzelnen Teilgebiete sehr unterschiedlich groß, entsprechend wurden einigen Teilgebieten sehr viele Forschungseinheiten zugeordnet, anderen nur wenige. Einige Forschungseinheiten aus Spezialgebieten der Chemie, wie etwa der, Geochemie' oder,Meereschemie', wurden keinem der Teilgebiete zugeordnet, aber dennoch mit bewertet. " (Bewertungsgruppe Chemie: $39 f$.)

In der Soziologie bereiten vor allem Unterschiede in Größe und Struktur der zu bewertenden Einheiten Schwierigkeiten. Außerdem erschwert die soziologische Publikationspraxis sogar die Abgrenzung des Faches selbst:

„Die Größenvarianz der gemeldeten Forschungseinheiten und die Varianz ihrer internen Heterogenität war insgesamt hoch, was in manchen Fällen die Bewertung auch bei Berücksichtigung ungleicher Mitgliederzahlen erschwerte. “(Ergebnisse Soziologie: 25)

„Hinzu kommt, dass die Soziologie disziplinär weniger deutlich abgegrenzt ist. So, wie sich die Mehrheit der Forschungseinheiten als interdisziplinär bezeichnet, erfolgt auch ein großer Teil der Publikationen von Soziologen nicht in eindeutig als soziologisch zu identifizierenden Zeitschriften, sondern in einem breiten Spektrum von über 1.000 Zeitschriften unterschiedlichster Spezialisierung und in Sammelwerken. “ (Steuerungsgruppe: 19)

Der Diskurs bemängelt regelmäßig die Unübersichtlichkeit der Forschung und die damit einhergehenden Abgrenzungs- und Zuschreibungsprobleme; Heterogenität und Komplexität der vorgefundenen Forschungs- und Publikationspraktiken werden immer wieder als Schwierigkeiten thematisiert. In dieser Problemdefinition zeigt sich eine weitere Facette normalistischer Prägung des Forschungsratings - und damit ein Verweis der technischen Dimension auf die zugrunde liegende Rationalität: Wo immer die normalistischen Techniken des Ratings auf Schwierigkeiten stoßen, wo immer die Vielfalt der sozialen Wirklichkeit sich den Normalisierungsbemühungen widersetzt, antwortet der Diskurs mit einer Problematisierung. Vielfalt und Unschärfe werden zum Problem erklärt - und zwar zum Problem für die betroffenen Forschungseinrichtungen. Denn der Bewertungsprozess wird trotz wahrgenommener Zuordnungs- und Abgrenzungsprobleme dennoch durchgeführt:

„Dieser Befund [der übermäßigen Heterogenität] dürfte sich auf viele andere geistes- und sozialwissenschaftliche Fächer [...] übertragen lassen. Die Ausweitung des Forschungsratings auf weitere dieser Fächer wird deshalb die Notwendigkeit mit sich bringen, Literaturdaten unter Mitwirkung der betroffenen Wissenschaftler zu korrigieren und in erheblichem Umfang neu zu erfassen. Der Zusammenhang mit der Leistungsbewertung stellt 
einen erheblichen Anreiz für Wissenschaftler eines Fachs dar, sich daran zu beteiligen." (Steuerungsgruppe: 38, eigene Hervorhebung)

Auf diese Weise wirken die Datenerhebungstechniken des Forschungsratings überall dort, wo sie die Komplexität der sozialen Wirklichkeit nicht bewältigen können, als Techniken der Sanktion: Sie sorgen dafür, dass Heterogenitätsüberschüsse tendenziell zu schlechteren Bewertungen führen und erzeugen damit Anreize für Selbst-Normalisierung. Oberflächlich betrachtet mögen die Datenerhebungstechniken des Forschungsratings den Verfahren gleichen, die in der Sozialwissenschaft zur Anwendung kommen; im Rahmen des Forschungsratings werden sie jedoch zu ,zwangsbewehrten Messverfahren' umfunktioniert und entfalten spezifische Machteffekte. Denn wo wissenschaftliche Datenerhebung bemüht ist, die Auswirkungen der Beobachtung auf das Handeln der Beobachteten zu minimieren, gilt hier gerade das Gegenteil: Das Forschungsrating maximiert die Reaktivität der Datenerhebungsverfahren indem es Beschreibung und Bewertung miteinander verschränkt. Heterogenitätsüberschüsse und Unschärfen führen schon im Rahmen der Datensammlung zu Nachteilen für die betroffenen Einrichtungen, die sich letztlich auch in der Bewertung niederschlagen. Abweichungen von der Normalitätsdefinition unterliegen damit einer quasi automatischen Sanktion, die in der Architektur des Verfahrens selbst enthalten ist und daher noch vor der Bewertung durch die Gutachter greift. Das Forschungsrating definiert auf diese Weise einen neuen Normalitätsstandard für die Wissenschaft, der beinhaltet, dass wissenschaftliche Leistung nur als solche anerkannt wird, wenn sie Kriterien der standardisierten Vermessbarkeit, Zählbarkeit, Zurechenbarkeit und Kalkulierbarkeit erfüllt (vgl. Angermüller 2010). Forschungseinrichtungen und Wissenschaftler werden dazu motiviert, diese Kriterien zu internalisieren.

\section{4. Subjekte: Adressaten und Universitäten}

Diskurse verweisen häufig auf Akteure und deren Eigenschaften und Absichten und spannen auf diese Weise Handlungsfelder auf, in denen verschiedene Figuren in bestimmten Beziehungen zueinander stehen. Diese diskursiv produzierten Figuren werden im Folgenden als „Subjekte“ bzw. „Subjekt-Formen“ bezeichnet. Indem Diskurse Subjekte auf bestimmte Weise formen und zueinander in Beziehung setzen, üben sie Einfluss auf diejenigen aus, die sich mit den angebotenen Subjektformen identifizieren. Im Diskurs des Forschungsratings spielen zwei Subjekte die Hauptrollen: Adressaten und Universitäten. Folgendes Zitat verdeutlicht die Rolle der Adressaten:

„Das Verfahren hilft damit, eine aus Gesellschaft und Politik an die Wissenschaft herangetragene Rechenschaftspflicht zu erfüllen. [...] Nachwuchswissenschaftler und fortgeschrittene Studenten aus dem In- und Ausland können mit Hilfe des Forschungsratings beurteilen, an welchen Einrichtungen ein passendes Umfeld für die ersten Schritte einer wissenschaftlichen Karriere besteht. In Fächern wie der Chemie erfüllt das Forschungsrating eine wichtige Orientierungsfunktion für potentielle Kooperationspartner aus der Industrie [...]. “ (Steuerungsgruppe: 28f.)

Studenten und Nachwuchswissenschaftler, Politiker und Entscheidungsträger der Wirtschaft sowie letztlich die gesamte Gesellschaft werden als hier Adressaten des Forschungsratings angeführt. Diese Adressaten sind als externe Nutzer und Förderer auf verschiedene Weise an Wissenschaft interessiert, jedoch steht diesem Interesse eine Überforderung durch die Komplexität und Heterogenität der Wissenschaft im Wege. Aus der Kombination von generellem Interesse und genereller Überforderung ergibt sich - so die diskursive Subjektbeschreibung ein allgemeines Bedürfnis nach einer normalisierenden Durcharbeitung der Wissenschaft. Wie weit dieses Interesse an Normalisierung trägt, macht folgende Passage deutlich: 
„Auch für Studierende oder Wissenschaftler aus dem Ausland [...] enthalten Rankings wertvolle Informationen, und es ist denkbar, dass sich die Verfügbarkeit solcher Auskünfte nicht nur auf die Ortswahl innerhalb eines Landes auswirkt, sondern die Interessenten sich überhaupt eher zu einem Gastaufenthalt in einem Land entschließen, wenn sie sich über die relative Qualität der zur Auswahl stehenden Institutionen gut informiert fühlen. " (Empfehlungen zu Rankings: 3)

Das Rating steigert die Attraktivität deutscher Universitäten nicht nur, indem es die Adressaten bei konkreten Entscheidungen unterstützt, sondern auch indem es einem generalisierten Bedürfnis nach Normalisierung entgegenkommt.

Im Diskurs spielen die Adressaten vor allem als Legitimationsinstrument eine Rolle. Sie treten dabei als Träger legitimer Interessen auf, die die Wissenschaft nicht ignorieren kann und darf. Durch die Anrufung der Adressaten und die Beschreibung ihrer Interessen entsteht im Diskurs ein Szenario, in dem das Rating-Verfahren wie selbstverständlich als notwendige und förderliche Maßnahme erscheint. Als personifizierte Letztbegründungen erscheinen die Adressaten im Diskurs überall dort, wo eine Entscheidung legitimiert, ein Zweifel ausgeräumt, einer Kritik vorgebeugt werden muss. Folgende Passagen illustrieren, wie vielfältig dieses Begründungswerkzeug eingesetzt wird:

„Für jedes Ranking muss die Objektmenge durch Benennung des Wissenschaftsbereichs, der betrachteten Institutionen sowie der Aggregationsebene der Bewertung festgelegt werden. Die Auswahl hängt von den Zielen und Adressaten ab. " (Empfehlungen zu Rankings: 36)

,Welche Kriterien verwendet werden, hängt von der Zielsetzung und den Interessen des jeweiligen Adressaten ab. “ (Empfehlungen zu Rankings: 39)

„,Der Träger eines Rankings sollte in erster Linie den Adressaten verantwortlich [...] sein. “ (Empfehlungen zu Rankings: 41)

„Der Wert der Ergebnisse des Forschungsratings für die Adressaten wird voraussichtlich steigen, wenn durch eine wiederholte Bewertung auch Trends erkennbar werden. " (Empfehlungen zum Forschungsrating: 11)

Wie man sieht, sind Adressaten und ihre Bedürfnisse ein bedeutendes Kriterium bei einer Vielzahl von Entscheidungen: Objekte, Fächer, Leistungsdimensionen und -kriterien, sogar die Trägerschaft des Ratings und die Frage danach, ob es in Zukunft wiederholt werden soll, müssen sich danach richten. Mithilfe dieser Strategie erreicht der Diskurs zwei Ziele: Erstens sorgt er für die Legitimation wichtiger Entscheidungen und externalisiert Begründungsprobleme. Hierin ist eine deutliche Verknüpfung zwischen der Subjekt- und der RationalitätsDimension zu sehen: Den Adressaten - die im Diskurs keine Stimme haben - wird einerseits ein Großteil der Begründungslast aufgebürdet; andererseits verstärkt die Beschwörung einer Vielzahl interessierter und bedeutende Beobachter den motivierenden Effekt des Sichtbarkeitsregimes. Zweitens propagiert der Diskurs hier eine spezifische Form der Selbstwahrnehmung für Politiker, Studenten und Wissenschaftler, die nahelegt, praktische Probleme auf die Unübersichtlichkeit der Wissenschaft zurückzuführen und künftige Entscheidungen an den Bewertungen des Forschungsratings auszurichten. Die im Forschungsrating verwendeten Sichtbarkeitsformen und Problematisierungen können auf diesem Weg auch außerhalb der Wissenschaft $\mathrm{Fu}$ fassen.

Die zweite bedeutende Subjekt-Form im Diskurs des Forschungsratings ist die Forschungseinrichtung bzw. die Universität. Das Besondere an dieser Subjekt-Form ist die Tatsache, dass der Diskurs hier eine Organisation mit einem Vokabular beschreibt, das üblicherweise nur bei Akteuren, d.h. bei handlungsfähigen, willensbegabten Individuen zum Einsatz kommt. Dass diese Redeweise im Diskurs des Forschungsratings auftaucht, ist keineswegs selbstverständ- 
lich; Forschungseinrichtungen könnten ebenso gut als bürokratische Gebilde, als soziale Arenen, als technisch-administrative Infrastrukturen oder als bildungs- und forschungspolitische Anstalten thematisiert werden. Stattdessen erscheinen sie als Subjekte mit quasi-akteurhaften Eigenschaften (vgl. Meier 2009). Dabei kommen zwei Spielarten der Akteurhaftigkeit vor: Einerseits wird als selbstverständlich vorausgesetzt, dass Forschungseinrichtungen, autonome Akteure' sind. Die Einrichtungen sind demnach Träger von Entscheidungen und Motivationen; sie haben ein eigenes Profil, das Stärken und Schwächen beinhaltet; sie sind lernfähig und können strategisch handeln, ja sie brauchen sogar Raum zum Ausdruck ihrer individuellen Einzigartigkeit:

,[E] in Forschungsrating von Universitäten und außeruniversitären Forschungseinrichtungen [...soll] diese bei strategischen Entscheidungen unterstützen. " (Empfehlungen zu Rankings: iii)

„,[Die Bewertungsgruppe] begrüßt ausdrücklich [...] das differenzierte Aufzeigen spezifischer Stärken und Schwächen einzelner Einrichtungen nach verschiedenen Kriterien, das vor allem den Einrichtungen selbst wichtige Erkenntnisse liefern kann und ihnen eine Basis bietet, ihre Profile weiterzuentwickeln. “ (Bewertungsgruppe Chemie: 6)

„Die Erfahrungen aus der Pilotstudie haben außerdem gezeigt, dass die möglichst präzise, aber zugleich nicht zu einengende Formulierung der Fragen für [...] die individuellen Ausdrucksmöglichkeiten der Einrichtungen [...] entscheidend ist. [Z] u eng definierte Fragen provozieren Kritik, wenn Einrichtungen glauben, sich nicht adäquat darstellen zu können. " (Bewertungsgruppe Chemie: 45f)

Auf der anderen Seite wird der Diskurs nicht müde, das Forschungsrating als Mittel zur Steigerung von Akteurhaftigkeit zu preisen: Forschungseinrichtungen ,können durch eine Bewertung in ihren Akteursqualitäten gestärkt werden.“ (Steuerungsgruppe: 34)

Insgesamt konstruiert der Diskurs die Forschungseinrichtungen und Hochschulen als Subjekte, deren Akteursqualitäten im Kern vorhanden, jedoch unvollständig ausgeprägt sind. Das Rating-Verfahren dient hier explizit als Instrument der Machtausübung, das Universitäten aus ihrem präreflexiven Halbschlaf erweckt und in vollwertige, rational-egoistisch motivierte Akteure verwandelt. Die Funktionsweise dieser Subjekt-Formierung ähnelt jener der Adressaten: Erstens geht es um die Legitimation des Ratings. Zweitens wird eine Subjekt-Form erzeugt, die den Einrichtungen als Normalisierungsschablone dienen kann, die ihnen ermöglicht und nahelegt, sich als strategische Akteure zu begreifen, die im Wettebewerb zueinander stehen und dem interessierten Blick von Adressaten ausgesetzt sind. Die diskursive Formierung und Relationierung dieser beiden Subjekt-Typen ist eine Praxis des Regierens, die sowohl beobachtete wissenschaftliche Akteure als auch externe, potenziell beobachtende Akteure mit spezifischen Selbstbeschreibungen, Weltbildern und Problemdefinitionen versorgt und auf diese Weise ihre Handlungsfelder strukturiert.

\section{Fazit}

Die Analyse hat gezeigt, dass das Forschungsrating nicht nur als eine spezifische Form der Regierung von Wissenschaft gedeutet werden kann, sondern darüber hinaus eine explizite Beschreibung, Erklärung und Bewertung dieser Regierungspraxis liefert und damit Ansätze einer Regierungskunst für die Wissenschaft formuliert. Sowohl panoptische als auch normalistische Elemente prägen diese Regierungskunst. Ihr zentraler Impetus zielt auf die Restrukturierung der Selbstwahrnehmung zentraler Akteure und Organisationen in der Wissenschaft, die sich in spezifischer Weise auf das Handeln der Akteure auswirken und letztlich die Funktionslogik wissenschaftlicher Selbststeuerung verändern soll. Sollte es dem Forschungsrating gelingen, sich dauerhaft im deutschen Wissenschaftssystem zu etablieren und prägend auf wissenschaftliche Akteure und Institutionen zu wirken, ist mit einer Reihe gravierender Ver- 
änderungen zu rechnen, die nicht auf der Vorderbühne wissenschaftlicher Selbstdarstellung haltmachen, sondern durch die Einpflanzung neuer Kriterien die wissenschaftliche Praxis tiefgreifend transformieren werden. Internationalität, Differenzierung, ökonomische Rationalität, standardisierte Erfassbarkeit und Formen akteurhafter Organisation werden im Rahmen des Forschungsratings zu eigenständigen Gütekriterien wissenschaftlicher Praxis.

In Bezug auf aktuelle Diagnosen zum Wandel der Wissenschaft zeigen diese Ergebnisse, dass das Forschungsrating über eine Ökonomisierung der Wissenschaft im Rahmen des ,akademischen Kapitalismus" hinausgeht (Rhoades / Slaughter 2004). Die Einführung ökonomischer Prinzipien ist zwar eindeutig Teil des Programms und das Interface ist eine soziale Formation, die vor allem in Märkten zu beobachten ist (Mützel 2009). Die vorliegenden Analysen zeigen jedoch, dass Ökonomisierung nur ein Teilaspekt eines umfassenden Prozesses ist, in dem die Normalisierung der Forschung eine bedeutende Rolle spielt. Erst im Zuge ihrer Normalisierung wird Forschung zu einem zählbaren, kalkulierbaren und bilanzierbaren Phänomen, das einer Ökonomisierung überhaupt zugänglich ist. Erst durch Normalisierung werden Forschungseinrichtungen auf eine Weise vergleichbar, die es ihnen ermöglicht, in marktförmige Konkurrenz zu treten. In ähnlicher Weise bestätigen die Ergebnisse zwar den Trend hin zur „unternehmerischen Universität“ (Maasen / Weingart 2008; Baumeler 2009). Darüber hinaus legen sie jedoch nahe, dass auch dies ein Teilaspekt einer umfassenderen Entwicklung ist, die Universitäten und Forschungsinstitute in vollwertige rationale Akteure verwandelt, die als Träger von Entscheidungen, Leistungen, Charaktereigenschaften und Erkenntnisprozessen taugen (vgl. Meier 2009). Die unternehmerische Universität ist eine Universität, die ihr Akteurspotenzial in bestimmter Weise nutzt; dass sie dieses Potenzial überhaupt besitzt, ist der Effekt einer am Panopticon orientierten Praxis des Regierens, die Universitäten zu handlungsfähigen Subjekten macht.

Da sich die vorgelegten Ergebnisse nur auf Interpretationen eines Diskurses stützen, drängt sich die Frage der Reichweite auf: Was spricht dafür, dass das Forschungsrating überhaupt dazu in der Lage ist, wissenschaftliche Praxis zu prägen? Schon die bedeutende gesellschaftliche Position des Diskursträgers legt jedoch nahe, dass der Diskurs des Forschungsratings nicht ungehört verhallen wird. Der Wissenschaftsrat ist eine zentrale Institution der deutschen Wissenschaftspolitik, die über große Reputation und Definitionsmacht verfügt. Zudem ist das Bewertungsverfahren bereits mit der wissenschaftlichen Praxis der beiden Disziplinen Chemie und Soziologie in Berührung gekommen und hat durch die Publikation der Ergebnisse in politische und öffentliche Debatten Einzug gehalten. Die Ausdehnung des Forschungsratings auf weitere Fächer und bereits angedachte Wiederholungen in den Pionierfächern werden die Wirkung auf die wissenschaftliche Praxis vertiefen und verstetigen, weil Lern- und Anpassungseffekte normal werden, während die Zonen noch nicht evaluierter Wissenschaft schrumpfen. Ein Blick in die Literatur zeigt, dass auch Autoren aus anderen Forschungstraditionen zu vergleichbaren Ergebnissen kommen. So ist etwa laut Wendy Espeland und Michael Sauder die standardisierte Vermessung und öffentliche Bewertung von Wissenschaft als reaktives Messverfahren zu betrachten, das systematisch das Handeln der betroffenen Akteure und Organisationen beeinflusst (Espeland / Sauder 2007). Zudem verläuft die Stoßrichtung des Forschungsratings - was etwa die Frage der Internationalität oder das Idealbild der akteurhaften Universität angeht - parallel zu anderen Reformen, allen voran der Exzellenzinitiative, die bekanntermaßen erhebliche Folgen für Universitäten und Forscher hat (Münch 2007; Hartmann 2009). Parallele Veränderungen umfassenderer Art beschreibt Michael Power als Entwicklung hin zur „Audit-Society“ (Power 2002). Demnach diffundieren Verfahren der Evaluation, Kontrolle und Rechenschaftsablage mithilfe quantifizierender Techniken in immer mehr gesellschaftliche Bereiche und werden zu selbstverständlichen Verifikationsritualen, die den gesellschaftlichen Umgang mit Vertrauen und Misstrauen regeln, während sie selbst uneingeschränktes, dabei aber kaum begründbares Vertrauen genießen. 
Das Forschungsrating ließe sich damit als wissenschaftsspezifischer Ausdruck eines umfassenden gesellschaftlichen Wandels betrachten. Als Manifestation einer neuen Regierungskunst im deutschen Wissenschaftssystem verdient es die besondere Aufmerksamkeit der wissenschaftlichen Gemeinschaft. Die soziologische Reflexion sollte sich nicht damit begnügen, es als „gegebenes Faktum“ zu nehmen und sich nicht auf die Frage beschränken, ob seine Bewertungen valide und reliabel, also letztlich gerecht sind, und wie sie sich dies sicherstellen ließe (Riordan / Ganser / Wolbring 2011: 149f). Als Praxis des Regierens von Wissenschaft beinhaltet das Forschungsrating eine Reihe diskussionswürdiger Konsequenzen, die nicht davon abhängen, wie der Bewertungsprozess im Einzelnen gestaltet ist: Wissenschaftler und Hochschulen, die mit Selbstnormalisierung und Effizienzsteigerung beschäftigt sind, haben weniger Ressourcen für Forschung und Lehre übrig. Unter Bedingungen verschärften Wettbewerbs bei ständiger externer Beobachtung können es sich nur arrivierte Forscher und prestigeträchtige Institute leisten, Neues auszuprobieren, was auf lange Sicht die Innovationskraft der Wissenschaft hemmen dürfte. Und nicht zuletzt unterstützt die diskursive Umwandlung der Hochschule zum akteurhaften Subjekt die Machtverschiebung von der akademischen Profession hin zur Organisation Universität (vgl. Schimank 2005). Die im Forschungsrating anvisierte Gestalt der Wissenschaft weist damit erhebliche Unterschiede zu etablierten Modellen akademischer Verhältnisse auf, wie sie etwa von Parsons (1973), Merton (1973) und Bourdieu $(1974,1998)$ formuliert wurden. Aus diesem Grund sollte das Forschungsrating möglichst offen, auch mit Blick auf seine Folgen für das soziale Gefüge der Wissenschaft, diskutiert werden.

\section{Literatur}

Angermüller, Johannes (2010): Wissenschaft zählen. Regieren im digitalen Panoptikum, in: Leviathan Sonderheft 25, S. 273-298.

Barthes, Roland (1966): Die strukturalistische Tätigkeit, in: Kursbuch 2, S. 190-196.

Baumeler, Carmen (2009): Entkopplung von Wissenschaft und Anwendung. Eine neo-institutionalistische Analyse der unternehmerischen Universität, in: Zeitschrift für Soziologie 38, S. 68-84.

Bourdieu, Pierre (1975): The specificity of the scientific field and the social conditions of the progress of reason, in: Social Science Information 14: S. 19-47.

Bourdieu, Pierre (1998): Vom Gebrauch der Wissenschaft. Für eine klinische Soziologie des wissenschaftlichen Feldes, Konstanz.

Dean, Mitchell (1995): Governing the unemployed self in an active society, in: Economy and Society 24 , S. 559-583.

Dean, Mitchell (1996): Putting the technological into government, in: History of the Human Sciences 47, S. 47-68.

Dean, Mitchell (2009): Governmentality. Power and rule in modern society, London.

Diaz-Bone, Rainer (2006): Zur Methodologisierung der Foucaultschen Diskursanalyse, in: FQS 7 / 1, abrufbar unter: http://www.qualitative-research.net/index.php/fqs/article/view/71/146, letztes Abrufdatum: 13.10.2011.

Diaz-Bone, Rainer (2007): Die französische Epistemologie und ihre Revisionen. Zur Rekonstruktion des methodologischen Standortes der Foucaultschen Diskursanalyse, in: FQS 8 / 2, abrufbar unter: http:// www.qualitative-research.net/index.php/fqs/article/view/238/528, letztes Abrufdatum: 13.10.2011.

Diaz-Bone, Rainer / Werner Schneider (2004): Qualitative Datenanalysesoftware in der sozialwissenschaftlichen Diskursanalyse - Zwei Praxisbeispiele, in: Reiner Keller / Andreas Hirseland / Werner Schneider / Willy Viehöver (Hrsg.), Handbuch Sozialwissenschaftliche Diskursanalyse, Wiesbaden: VS Verlag für Sozialwissenschaften, S. 457-494.

Espeland, Wendy / Michael Sauder (2007): Rankings and Reactivity: How Public Measures Recreate Social Worlds, in: American Journal of Sociology 113, S. 1-40. 
Ewald, François (1990): Norms, Discipline, and the Law, in: Representations, S. 138-161.

Foucault, Michel (1974): Die Ordnung des Diskurses, München.

Foucault, Michel (1982): The Subject and Power, in: Hubert L. Dreyfus / Paul Rabinow (Hrsg.), Michel Foucault: beyond structuralism and hermeneutics, Brighton, S. 208-226.

Foucault, Michel (2006): Die Geburt der Biopolitik. Vorlesung am Collège de France, 1978 - 1979. Frankfurt / Main.

Gerhards, Jürgen / Gert G. Wagner (2008): Es geht um die Qualität. Was ist das Besondere an dem Forschungsrating des Wissenschaftsrates und warum sollte es Schule machen?, in: Forschung \& Lehre 14, S. 532-533.

Hartmann, Michael (2009): Die Exzellenzinitiative und ihre Folgen, in: Leviathan 38: S. 369-387.

Jäger, Margarete / Siegfried Jäger (2007): Deutungskämpfe. Theorie und Praxis Kritischer Diskursanalyse, Wiesbaden.

Laudel, Grit / Jochen Gläser (2006): Tensions between evaluations and communication practices, in: Journal of Higher Education Policy and Management 28, S. 289-295.

Maasen, Sabine / Peter Weingart (2008): Unternehmerische Universität und neue Wissenschaftskultur, in: Hildegard Matthies / Dagmar Simon (Hrsg.), Wissenschaft unter Beobachtung. Effekte und Defekte von Evaluationen, Wiesbaden, S. 141-160.

Mathiesen, Thomas (1997): The Viewer Society: Michel Foucault's 'Panopticon' Revisited, in: Theoretical Criminology 1: S. 215-234.

Meier, Frank (2009): Die Universität als Akteur. Zum institutionellen Wandel der Hochschulorganisation, Wiesbaden.

Merton, Robert K. (1973): The sociology of science. Theoretical and empirical investigations, Chicago.

Münch, Richard (2007): Die akademische Elite. Zur sozialen Konstruktion wissenschaftlicher Exzellenz, Frankfurt / Main.

Münch, Richard (2009): Die Konstruktion soziologischer Exzellenz durch Forschungsrating, in: Soziale Welt 60, S. 63-90.

Münch, Richard / Baier, Christian (2009): Die Konstruktion der soziologischen Realität durch Forschungsrating, in: Berliner Journal für Soziologie 19, S. 295-319.

Mützel, Sophie (2009): Geschichten als Signale: Zur diskursiven Konstruktion von Märkten., in: Rainer Diaz-Bone / Gertraude Krell (Hrsg.), Diskurs und Ökonomie. Diskursanalytische Perspektiven auf Märkte und Organisationen, Wiesbaden, S. 225-244.

Neidhardt, Friedhelm (2006 a): Fehlerquellen und Fehlerkontrollen in den Begutachtungssystemen der Wissenschaft, in: Stefan Hornbostel / Dagmar Simon (Hrsg.), Wie viel (In-)Transparenz ist notwendig? Peer Review Revisited, Bonn, S. 7-14.

Neidhardt, Friedhelm (2006 b): Forschungsrating der deutschen Soziologie durch den Wissenschaftsrat, in: Soziologie 35, S. 303-308.

Parsons, Talcott / Gerald M. Platt (1973): The American university, Cambridge.

Power, Michael (2002): The audit society. Rituals of verification, Oxford.

Rhoades, Gary / Sheila Slaughter (2004): Academic Capitalism in the New Economy: Challenges and Choices, in: American Academic 1, S. 37-59.

Schimank, Uwe (2005): 'New Public Management' and the Academic Profession: Reflections on the German Situation, in: Minerva 43, S. 361-376.

Schwab-Trapp, Michael (2004): Methodische Aspekte der Diskursanalyse. Probleme der Analyse diskursiver Auseinandersetzungen am Beispiel der deutschen Diskussion über den Kosovokrieg, in: Reiner Keller / Andreas Hirseland / Werner Schneider / Willy Viehöver (Hrsg.), Handbuch Sozialwissenschaftliche Diskursanalyse, Wiesbaden: VS Verlag für Sozialwissenschaften, S. 169-196. 
Strauss, Anselm / Juliet Corbin (1999): Grounded theory. Grundlagen qualitativer Sozialforschung, Weinheim.

White, Harrison C. (2008): Identity and control. How social formations emerge, Princeton.

Wissenschaftsrat, 2004: Empfehlungen zu Rankings im Wissenschaftssystem. Teil 1: Forschung, Hamburg, abrufbar unter: http://www.wissenschaftsrat.de/texte/6285-04.pdf, letztes Abrufdatum: 13.10.2011.

\section{Verzeichnis der Primärtexte}

Wissenschaftsrat (2004): Empfehlungen zu Rankings im Wissenschaftssystem. Teil 1: Forschung, Hamburg, abrufbar unter: http://www.wissenschaftsrat.de/texte/6285-04.pdf, letztes Abrufdatum: 7.8.2009.

Wissenschaftsrat (2007): Forschungsleistungen deutscher Universitäten und außeruniversitärer Einrichtungen in der Chemie, Köln, abrufbar unter: http://www.wissenschaftsrat.de/texte/pilot_ergeb_chemie.pdf, letztes Abrufdatum: 7.8.2009.

Wissenschaftsrat (2008): Pilotstudie Forschungsrating Chemie. Abschlussbericht der Bewertungsgruppe, Köln, abrufbar unter: http://www.wissenschaftsrat.de/texte/8370-08.pdf, letztes Abrufdatum: 7.8.2009.

Wissenschaftsrat (2008): Pilotstudie Forschungsrating Soziologie. Abschlussbericht der Bewertungsgruppe, Köln, abrufbar unter: http://www.wissenschaftsrat.de/texte/8422-08.pdf, letztes Abrufdatum: 7.8.2009.

Wissenschaftsrat (2008): Forschungsleistungen deutscher Universitäten und außeruniversitärer Einrichtungen in der Soziologie, Köln, abrufbar unter: http://www.wissenschaftsrat.de/texte/pilot_ergeb_sozio.pdf, letztes Abrufdatum: 7.8.2009.

Wissenschaftsrat (2008): Bericht der Steuerungsgruppe zur Pilotstudie Forschungsrating Chemie und Soziologie, Köln, abrufbar unter: http://www.wissenschaftsrat.de/texte/8453-08.pdf, letztes Abrufdatum: 7.8.2009.

Wissenschaftsrat (2008): Empfehlungen zum Forschungsrating, Rostock, abrufbar unter: http://www.wissenschaftsrat.de/texte/8485-08.pdf, letztes Abrufdatum: 7.8.2009.

Christian Baier

Otto-Friedrich-Universität Bamberg Lehrstuhl für Soziologie II

Lichtenhaidestr. 11 christian.baier@uni-bamberg.de 